\title{
MRI reveals slow clearance of dead cell transplants in mouse forelimb muscles
}

\author{
YANHUI ZHANG ${ }^{1,2^{*}}$, HONGYAN ZHANG ${ }^{2,3^{*}}$, LIJUN DING $^{4}$, HAILU ZHANG ${ }^{2}$, \\ PENGLI ZHANG ${ }^{1,2}$, HAIZHEN JIANG ${ }^{1}$, BO TAN $^{2}$ and ZONGWU DENG ${ }^{2}$
}

\author{
${ }^{1}$ College of Sciences, Shanghai University, Shanghai $200444 ;{ }^{2}$ CAS Key Laboratory of Nano-Bio Interface and \\ Division of Nanobionics Research, Suzhou Institute of Nano-Tech and Nano-Bionics, Chinese Academy of Sciences, \\ Suzhou, Jiangsu 215123; ${ }^{3}$ Institute of Environmental and Chemical Engineering, Shanghai University, \\ Shanghai 200444; ${ }^{4}$ Center for Reproductive Medicine, Nanjing Drum Tower Hospital, \\ Nanjing University Medical School, Nanjing, Jiangsu 210008, P.R. China
}

Received February 3, 2017; Accepted July 7, 2017

DOI: $10.3892 / \mathrm{mmr} .2017 .7100$

\begin{abstract}
A small molecule tetraazacyclododecane-1,4,7,10tetraacetic acid (Gd-DOTA) ${ }_{4}$-TPP agent is used to label human mesenchymal stem cells (hMSCs) via electroporation (EP). The present study assessed the cytotoxicity of cell labeling, in addition to its effect on cell differentiation potential. There were no significant adverse effects on cell viability or differentiation induced by either EP or cellular uptake of (Gd-DOTA) $)_{4}$-TPP. Labeled live and dead hMSCs were transplanted into mouse forelimb muscles. $\mathrm{T}_{2}$-weighted magnetic resonance imaging (MRI) was used to track the in vivo fate of the cell transplants. The labeling and imaging strategy allowed long term tracking of the cell transplants and unambiguous distinguishing of the cell transplants from their surrounding tissues. Cell migration was observed for live hMSCs injected into subcutaneous tissues, however not for either live or dead hMSCS injected into limb muscles. A slow clearance process occurred of the dead cell transplants in the limb muscular tissue. The MRI results therefore reveal that the fate and physiological activities of cell transplants depend on the nature of their host tissue.
\end{abstract}

\section{Introduction}

Magnetic resonance imaging (MRI) is thought to be one of the most promising visualization tools for in vivo tracking of

Correspondence to: Dr Zongwu Deng or Dr Bo Tan, CAS Key Laboratory of Nano-Bio Interface and Division of Nanobionics Research, Suzhou Institute of Nano-Tech and Nano-Bionics, Chinese Academy of Sciences, 398 Ruoshui Road, Suzhou Industrial Park, Suzhou, Jiangsu 215123, P.R. China

E-mail: zwdeng2007@sinano.ac.cn

E-mail: btan2012@sinano.ac.cn

*Contributed equally

Key words: MRI, (Gd-DOTA) $)_{4}$-TPP, cell transplants, hMSCs, mouse limb muscle transplanted stem cells in terms of their viability, migration and homing, response to various endogenous stimuli (1-4). MRI tracking of stem cells requires labeling of the cells with contrast agents to allow them distinguished from in vivo tissues. Cells have been labeled with superparamagnetic iron oxide nanoparticles (SPIONs), Gd-chelates of different structures, and many other agents to yield information on in vivo cell viability, migration and differentiation (1-4). In addition to cell labeling, MR image interpretation of cell transplants also requires an in-depth understanding of its in vivo physiology, particularly in terms of cell viability, release and clearance of MR contrast agents, clearance of dead cell transplants, etc. in specific tissues.

For example, to address the issue of in vivo viability of exogenous cells, Khurana et al (5) described a strategy that can report death of cell transplants at arthritic joint. The strategy involves preloading macrophages in the reticuloendothelial system with SPIONs via intravascular injection and recruit of the SPIONs-labeled macrophages to the site of dead cell transplants so that a dark contrast results at the site (6). Later, Nejadnik et al developed a caspase activatable Gd agent for report of stem cell death in arthritic joints (7). They developed a caspase-3-sensitive MRI probe which self-assembles into nanoparticles upon hydrolysis by caspase- 3 released by dead cell transplants so that a signal enhancement/bright contrast results at the site. Ngen et al used a dual-contrast method to image cell transplants that can also report cell death (8). The strategy includes preloading stem cells with both SPIONs and Gd-DTPA so that the cells appear in dark contrast after transplantation. Dead cells release Gd-DTPA faster than SPIONs, and the released Gd-DTPA diffuses away and induces a signal enhancement around the dead cell transplant. These strategies reveal information on cell death but no information on in vivo fates of live cell transplants. Nevertheless, tracking of live cells is more important for understanding their functions and evaluating clinical benefits of cell transplantation (9).

In vivo detachment of MR contrast agents from labeled cells and its subsequent fate is a critical issue for MR image interpretation as addressed by several groups (8-15). This process usually depends on the molecular size of the agents 
and viable status of the cells. Release of small molecule agents is believed to be faster than large molecule agents or nanoparticles (8). Difference in release rate and mechanism between live and dead cells is expected but has not yet been addressed in detail. For example, macrophage uptake of released SPIONs has been reported (12-15), which may lead to overestimation of cell viability or image misinterpretation. The in vivo clearance process and mechanisms of dead cell transplants and its dependence on the nature of its host tissue also remains an issue to be addressed.

Recently, we have reported that labeling cells via electroporation (EP) with a small molecule (Gd-DOTA) i $_{\mathrm{i}}$ TPP $(i=1,2,4)$ agent induces its clustering on cell membrane and subsequent formation of cell-assembled vesicles containing the clustered agents. The labeling strategy allows long term tracking of intracranial transplants of labeled cells under $\mathrm{T}_{2}$-weighted MRI and reveals abundant information on in vivo fates of the cell transplants (16). In this work, we further use this labeling and imaging strategy to track cell transplants in mice limb muscles. Cell transplantation into mice limb has been used to evaluate the therapeutic effect of stem cells on ischemic tissues (17-23). However, the blood flow recovery resulting from these treatments does not always appear to be satisfactory $(20,23)$, the cause of which is usually ascribed to the death of transplanted cells before they can exert therapeutic effects. In this respect, Yamaoka and coworkers have developed a PVA-Gd-DOTA conjugates to label mesenchymal stem cells (MSCs) for transplantation into a rat model of hindlimb ischemia and have claimed that $\mathrm{T}_{1}$-weighted MRI can provide information on cell survival $(9,15,23)$. Nevertheless, additional information is required to distinguish dead cells from live ones. In addition, the molecular weight of the PVA is large $(\sim 75,000 \mathrm{kDa})$ so that it still takes days to clear the agents in vivo (23). Here we use a small molecule (Gd-DOTA) -TPP $_{4}$ agent to bypass the slow clearance of MR contrast agent and demonstrate slow clearance of dead cell transplants in mouse forelimb muscles.

\section{Materials and methods}

Chemicals were purchased from Sinopharm Chemical Reagent Co., Ltd. (Shanghai, China). All chemicals are of analytical grade and were used as received without further purification unless otherwise stated. Milli-Q water $\left(18.2 \mathrm{M} \Omega \mathrm{cm}^{-1}\right)$ was used throughout the experiments.

Structure of (Gd-DOTA $)_{4}$-TPP. (Gd-DOTA $)_{4}$-TPP is used as the MRI probe in this study. The chemical structure of

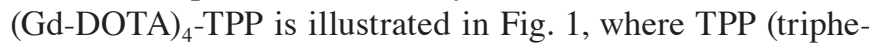
nylphosphonium) is a positively charged biomarker that can bind specifically with mitochondria $(24,25)$. When it is coupled with single or multiple Gd-DOTA via a mediation moiety, it can function as an MRI probe (16). Details on synthesis and characterization of the probe can be found elsewhere (16).

Culture of human MSCs (hMSCs). hMSCs were obtained as a generous gift from Dr Jianwu Dai at Institute of Genetics and Developmental Biology, Chinese Academy of Sciences. hMSCs were cultured at $37^{\circ} \mathrm{C}$ in a $5 \% \mathrm{CO}_{2}$ incubator $(3111$; Thermo Fisher Scientific, Inc., Waltham, MA, USA) in DMEM

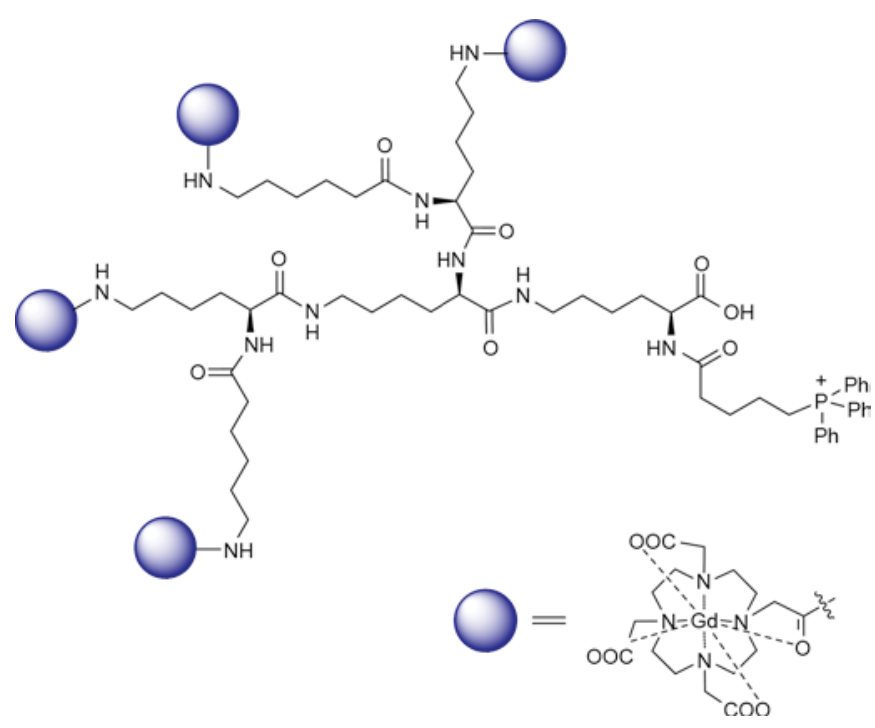

Figure 1. Schematic chemical structure of (Gd-DOTA) $)_{4}$-TPP used in this study. TPP was coupled with four Gd-DOTA via a mediation moiety made of lysine.

F-12 medium with $10 \%$ FBS and $1 \%$ penicillin-streptomycin. All cell culture related reagents were purchased from Gibco (Grand Island, NY, USA).

Cell labeling with $(\mathrm{Gd}-\mathrm{DOTA})_{4}$-TPP. hMSCs were seeded into $100 \times 20 \mathrm{~mm}$ style cell culture dishes at a density of approximately $1 \times 10^{6}$ cells per dish and maintained for $24 \mathrm{~h}$. Cells were trypsinized, and centrifuged at 1,000 rpm for $5 \mathrm{~min}$. The precipitated cells were re-suspended in $200 \mu$ l EP-buffer in the absence or presence of (Gd-DOTA) $)_{4}$ TPP at probe concentrations between $0 \sim 2.0 \mathrm{mM}$, and were transferred to 96-well plates. Six electrical pulses of $100 \mu \mathrm{s}$ at $\sim 120 \mathrm{~V}$ and an interval of $1 \mathrm{~s}$ were then applied to the cells using X-Porator ${ }^{\circledR}$ EBXP-H1 (Suzhou Etta Biotech Co., Ltd., Suzhou, China). After EP-labeling, the cells were collected and suspended in $4 \mathrm{ml}$ DMEM-F12. EP-labeling with (Gd-DOTA) $)_{4}$-TPP results in its clustering on cell membrane followed by cell assembly of intracellular vesicles containing the clustered agent (16). After the completion of labeling, the hMSCs were rinsed three times with 2-3 ml PBS to remove the residual materials followed by cell culture for an additional 2 3 days prior to cell transplantation. The adherent cells were collected by treating with trypsin.

Cytotoxicity assessment of (Gd-DOTA $)_{4}$ TPP on hMSCs. For assessment of cytotoxicity induced by EP or cellular uptake

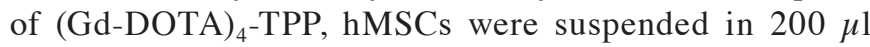
elecroporation (EP)-buffer containing 0, 0.5, 1.0, 2.0 mM of (Gd-DOTA) $)_{4}$-TPP, respectively. Defined electrical pulses were applied to the cells. After EP-labeling, the cells were collected and suspended in $4 \mathrm{ml}$ DMEM-F12, rinsed three times with 2-3 ml PBS, followed by transferred into a 96-well plate. In vitro cell viability of (Gd-DOTA) $)_{4}$-TPP-labeled hMSCs was assessed by using a standard MTT cytotoxicity assay. Apoptosis and necrosis of hMSCs induced by (Gd-DOTA) - $_{4}$ TPP was assessed immediately after EP-labeling using an apoptosis and necrosis assay kit (BD Pharmingen, San Diego, CA, USA). Single-cell suspensions were analyzed by FACS within $1 \mathrm{~h}$. 
Assessment of the differentiation potential of hMSCs after labeling. Upon $80 \%$ confluence, hMSCs at passage 4 were trypsinized and suspended in $200 \mu \mathrm{l} \mathrm{EP-buffer}$ containing 0, $2.0 \mathrm{mM}$ of (Gd-DOTA) ${ }_{4}$-TPP, respectively. Then defined electrical pulses were applied to the cells. After EP-labeling, the cells were collected and suspended in $4 \mathrm{ml}$ DMEM-F12, followed by gentle washes with PBS for three times to remove unbound (Gd-DOTA) ${ }_{4}$-TPP. Control experiments without EP-labeling were also performed. hMSCs were re-plated onto 24-well plates with defined glass coverslips in the wells. The seeding hMSCs were cultured overnight in $2 \mathrm{ml} \mathrm{DMEM-F12}$ to allow cell attachment.

For adipogenic differentiation, hMSCs were treated with adipogenic differentiation medium consisting of $1 \mu \mathrm{M}$ dexamethasone, $5 \mu \mathrm{g} / \mathrm{ml}$ insulin, $0.5 \mathrm{mM}$ isobutyl-methylxanthine (IBMX) and $60 \mu \mathrm{M}$ indometacin in the DMEM medium for up to 14 days. Lipid vacuole formation in hMSCs was detected by Oil-Red O staining. Briefly, cultures were fixed in 4\% paraformaldehyde for $30 \mathrm{~min}$, and then stained in $0.36 \%$ Oil-Red O for $30 \mathrm{~min}$. After washed completely with $60 \%$ isopropanol once and with PBS three times, adipogenic cells were imaged by Nikon Ti-E microscopy. For osteogenic differentiation, hMSCs were treated with osteogenic supplements containing $100 \mathrm{nM}$ dexamethasone, $0.05 \mathrm{mg} / \mathrm{ml}$ ascorbic acid, and $0.01 \mathrm{M} \beta$-glycerophosphate in the DMEM medium for up to 21 days with medium change every 3-4 days. Differentiation of hMSCs into osteogenic cells was determined by alizarin red staining. Adipogenic and osteogenic differentiation of hMSCs was quantified by measuring the absorbance of Oil-Red $\mathrm{O}$ and alizarin red extracted from cell lysates at $490 \mathrm{~nm}$, respectively (26).

In vivo $M R$ imaging of (Gd-DOTA $)_{4}$-TPP-labeled hMSCs. Animal handling was carried out at the Animal Laboratory of Soochow University (Suzhou, China). All animal experiments were conducted in accordance with the university's guidelines and were approved by the university's ethics committee.

(Gd-DOTA) $)_{4}$-TPP-labeled hMSCs were harvested by trypsinization and suspended in PBS. Male nude mice (4-6 weeks old) of 18-20 g body weight were anesthetized by intraperitoneal injection of $10 \%$ chloral hydrate solution at a dose of $5 \mathrm{ml} / \mathrm{kg}$ body weight before fixed in a stereotaxic apparatus. Defined number of labeled hMSCs in $5 \mu \mathrm{l}$ PBS was injected into the muscle of mouse forelimbs at a rate of $0.5 \mu \mathrm{l} / \mathrm{min}$ by using a Hamiltion microsyringe with a 27 -gauge needle. The needle was left in place for an additional $5 \mathrm{~min}$ before withdrawal to reduce the protraction of the cells into the needle track. Cell transplantation was conducted on the right forelimb. The left forelimb without cell injection can be taken as a control. The nude mice were then transferred and fixed into an animal handling system coupled with a Micromouse RF probe. Afterwards, they were inserted into the gradient system in an 11.7 T micro-imaging system (Bruker Corp., Ettlingen, Germany). During image acquiring, the mice were monitored by a life monitoring facility. Cell transplantation was conducted at mouse forelimbs because the Micromouse probe allows scan of a mouse only above its waist.

The cell transplants labeled with (Gd-DOTA) ${ }_{4}$-TPP were then tracked by in vivo $\mathrm{T}_{2}$-weighted $\mathrm{MRI}$ at $11.7 \mathrm{~T}$. In vivo $\mathrm{T}_{2}$-weighted images were acquired by using RARE sequence with TR/effective TE $=3000 / 22 \mathrm{msec}$, number of average $=8$, FOV $=2.0 \times 2.0 \mathrm{~cm}^{2}$, matrix $=128 \times 128$ with slice thickness of $0.5 \mathrm{~mm}$. The corresponding voxel size is $156 \times 156 \times 500 \mu \mathrm{m}^{3}$. The in vivo MRI experiments were terminated on day 9 after cell transplantation.

Histological analysis for cell apoptosis. After receiving injection of $\sim 3 \times 10^{5}$ hMSCs EP-labeled with (Gd-DOTA) $)_{4}$-TPP into the forelimb muscle, mice were sacrificed on day 0,1 , 2, 4, 7 and 10 after cell transplantation and in vivo MRI. The muscular tissues with cell transplants were collected for the following procedures with guide of in vivo MR images. The samples were first fixed in $4 \%$ paraformaldehyde for $4 \mathrm{~h}$ and then soaked in $20 \%$ sucrose solutions overnight. Muscular tissues were then embedded into OCT (Leica, Nussloch, Germany) and frozen at $-30^{\circ} \mathrm{C}$ for $5-10 \mathrm{~min}$. The frozen tissues were then continuously sectioned to $7 \mu \mathrm{m}$ thickness using a cryostat (Leica). The sections were adhered to slides treated by $0.1 \%$ polylysine. Slides were stained with primary antibody of cleaved caspase-3 (1:400, 9661; Cell Signaling Technology, Danvers, MA, USA). After washing with PBS for three times, sections were incubated with secondary antibody of goat anti-rabbit IgG H\&L (Alexa Fluor ${ }^{\circledR}$ 555; Abcam, Cambridge, UK). After mounted with ProLong ${ }^{\circledR}$ gold antifade reagent with DAPI (Invitrogen Life Technologies, Carlsbad, CA, USA), histological analysis and expression of fluorescence was observed immediately under confocal fluorescence microscopy (TCS SP8; Leica). Apoptotic cells appear in red with C-caspase-3 staining. Nuclei of either live or apoptotic cells appear in blue with DAPI staining.

Statistical analysis. Numerical values are expressed as the mean \pm standard deviation. Each experiment was repeated three times. Statistical significance was evaluated using t-test ANOVA analysis. $\mathrm{P}<0.05$ was considered to indicate a statistically significant difference.

\section{Results and Discussion}

Cytotoxicity of (Gd-DOTA $)_{4}$-TPP on hMSCs. The cell viability of hMSCs after EP-labeling with (Gd-DOTA) - $_{4}$-TPP was assessed by the MTT assay. Cell damage can be induced by either EP or cellular uptake of (Gd-DOTA) ${ }_{4}$-TPP or both. EP is a convenient means to introduce nonspecifically various substrates into the cytoplasma of many kinds of cells $(15,23)$. However, cells might be damaged by EP. The damage can be mitigated by using mild conditions as in the current work. Fig. 2A depicts cell viability of control hMSCs, and hMSCs subjected to EP in the absence or presence of

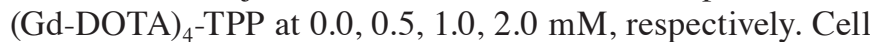
viability of hMSCs subjected to EP is above $90 \%$ of that of control hMSCs in all investigated cases. Fig. 2B depicts the apoptosis and necrosis of hMSCs induced by EP or cellular uptake of (Gd-DOTA) ${ }_{4}$-TPP. The normal cells represent $\sim 92 \%$ of total cells for control hMSCs, and 86 90\% for hMSCs subjected to EP. No obvious difference exhibits among hMSCs subjected to EP in the absence or presence of (Gd-DOTA $)_{4}$-TPP. These results suggest that both the EP conditions and the cellular uptake of (Gd-DOTA) $)_{4}$-TPP are tolerable by the hMSCs. 

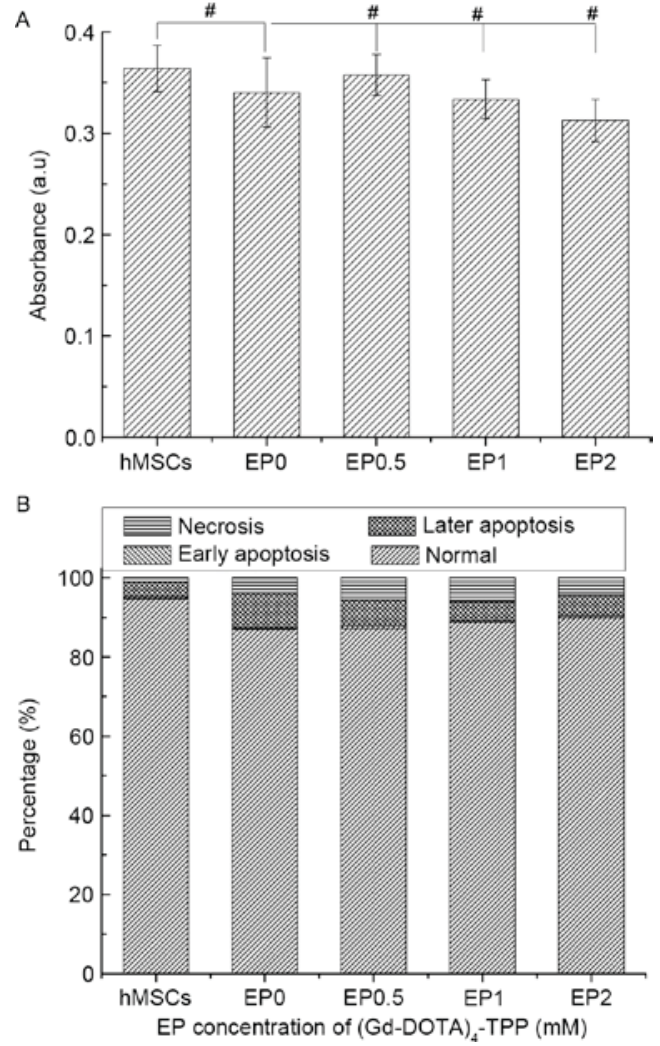

Figure 2. (A) In vitro cell viability and (B) apoptosis and necrosis of control human mesenchymal stem cells (hMSCs), hMSCs subjected to electroporation (EP) in the absence or presence of (Gd-DOTA) $)_{4}$-TPP. Numbers behind EP indicate EP concentration of (Gd-DOTA $)_{4}$-TPP. " ${ }^{*}$ indicates no significant difference.

Differentiation potential of hMSCs after labeling. The effect of EP and cellular uptake of (Gd-DOTA) ${ }_{4}$ TPP on the differentiation potential of hMSCs is also a concern for developing stem cell tracking probes. We explored possible adverse effects of EP-labeling with (Gd-DOTA) $)_{4}$-TPP on differentiation of hMSCs into adipocytes and osteocytes. For adipogenic differentiation, hMSCs EP-labeled with (Gd-DOTA) $)_{4}$-TPP were treated with adipogenic supplements for 14 days. Red lipid vacuole was clearly observed from Oil-red O staining of both differentiated control hMSCs (Fig. 3A/a2) and hMSCs subjected to EP in the absence (Fig. 3A/a3) or presence (Fig. 3A/a4) of (Gd-DOTA) $)_{4}$ TPP with respect to undifferentiated control hMSCs (Fig. 3A/a1). Quantification of Oil-Red O extracted from adipogenic cells at $490 \mathrm{~nm}$ absorbance revealed similar levels of adipogenic differentiation between hMSCs subjected to EP in the absence or presence

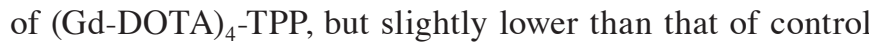
hMSCs (Fig. 3C). For osteogenic differentiation, the cells were treated with osteogenic supplements for 21 days, and detected by alizarin red staining (Fig. 3B). Similar differentiation capability exhibits for both differentiated control hMSCs (Fig. 3B/b2) and hMSCs subjected to EP in the absence (Fig. 3B/b3) or presence (Fig. 3B/b4) of (Gd-DOTA) ${ }_{4}$-TPP with respect to undifferentiated control hMSCs (Fig. 3B/b1), as revealed also by colorimetric quantification of alizarin red extracted from osteogenic cells (Fig. 3D). The results suggest that EP-labeling may exert some adverse effects on differentiation of hMSCs into adipocytes and osteocytes but no additional adverse effects are originated from cellular uptake of (Gd-DOTA $)_{4}$-TPP.

In vivo MR imaging of (Gd-DOTA) ${ }_{4}$-TPP-labeled hMSCs. Figs. 4 and 5 present $\mathrm{T}_{2}$-weighted MRI images of cell transplants of live and dead hMSCs EP-labeled with (Gd-DOTA) ${ }_{4}$-TPP as a function of post-transplantation time, respectively. The images reveal abundant information on in vivo fates of the cell transplants. We first note that significant signal reduction (dark contrast) exhibits at all injection sites immediately after cell transplantation [Figs. 4 (S1-S6 and 0 day) and 5 (S1-S5 and 0 day), arrows]. The signal reduction effect can persist over 9 days for both live [Fig. 4 (S2-S6 and 0-9 days)] and dead [Fig. 5 (S1-S5 and 0-9 days)] cell transplantation.

While Gd agents have long been used as $T_{1}$ contrast agents because they usually induce a signal enhancement effect in $\mathrm{T}_{1}$-weighted MRI, we have demonstrated that cell labeling via EP induces clustering of (Gd-DOTA $)_{\mathrm{i}}$-TPP agent on cell membrane which is subsequently followed by the formation of cell-assembled nanoclusters in the cytoplasm (16). As a consequence, two distinct cellular distributions of (Gd-DOTA $)_{4}$-TPP result from this labeling strategy: Freely and evenly distributed in the cytosol or (Gd-DOTA) $)_{4}$-TPP nanoclusters in the cytoplasm. The former exhibits fast release via exocytosis. The latter, on one hand, significantly promotes the intracellular retention time of the (Gd-DOTA) $)_{\mathrm{i}}$-TPP agent. On the other hand, it enormously reduces the cellular longitudinal relaxivity of the (Gd-DOTA) $)_{i}$ TPP agent, i.e., its acceleration effect on $T_{1}$-relaxation of cellular water protons so that a significant MR signal reduction effect exhibits. As a result, combination

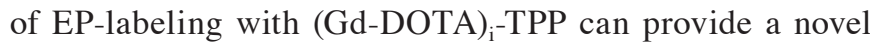
tool for long term in vivo tracking of cell transplants under $\mathrm{T}_{2}$-weighted MRI (16).

Second, we note that the dark contrast of the dead cell transplants persists over 9 days with neither obvious migration from the cell injection site nor obvious size shrinkage (Fig. 5, purple arrows). We have previously demonstrated that (Gd-DOTA $)_{\mathrm{i}}$-TPP induces signal reduction only when it is clustered and contained in intracellular vesicles, whereas (Gd-DOTA) $)_{4}$ TPP released as a result of exocytosis of live cells or membrane rupture of dead cells will induce a signal enhancement in the surrounding tissue (16). The long-time persistence of the dark contrast at the cell injection site thus indicates a slow clearance process of dead cells in the muscular tissue. This observation is also in agreement with our results on intracranial cell transplantation (16). A subcutaneous part of the dead cell transplant can also be observed and is slowly cleared out, as indicated by red arrows in S5 and 0-9 days of Fig. 5 .

The MRI results of live cell transplantation (Fig. 4) indicates the formation of one cell clot in the limb muscle (S1-S4 and 0-9 days, purple arrows) and several others in the subcutaneous tissues between the limb and body (Fig. 4: S1-S4 and 0 day, blue arrows; S5-S6 and 0-9 days, red arrows) after cell transplantation. These cell clots exhibit quite different fates in the subsequent development. The cell clot in the limb muscle appears similar to that of the dead cell transplant, both persisting over 9 days with no obvious change in either contrast or clot size. No obvious signal enhancement effect is induced in its surrounding muscular tissue. The cell clot indicated by blue arrows in S1-S4 and 0 day of Fig. 4 induces 

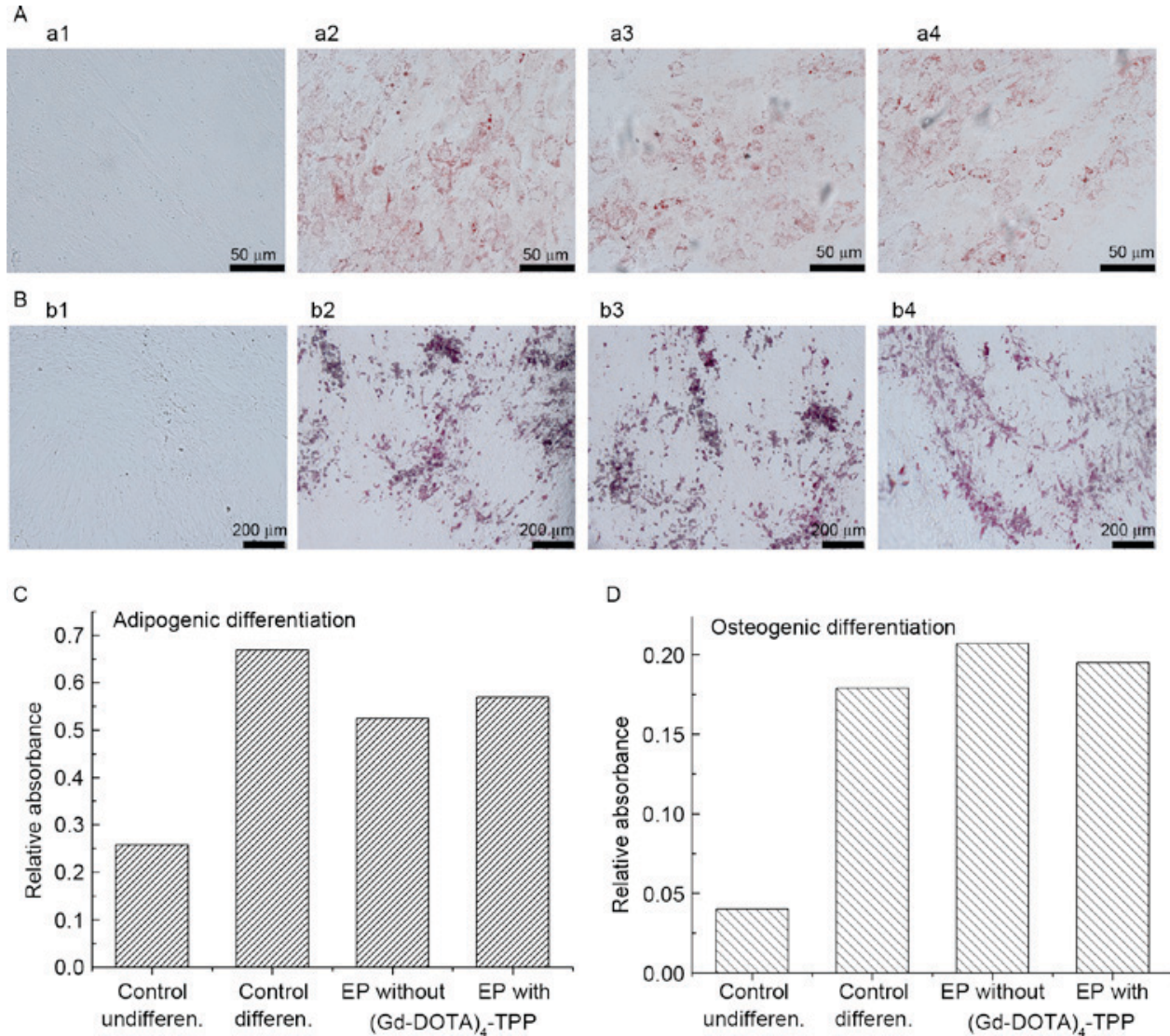

Figure 3. (A and C) Adipogenic and (B and D) osteogenic differentiation potential of (a1/b1) non-differentiated control human mesenchymal stem cells (hMSCs), (a2/b2) differentiated control hMSCs, (a3/b3) differentiated hMSCs subjected to EP in the absence or (a4/b4) presence of (Gd-DOTA) -TPP. EP, electroporation.

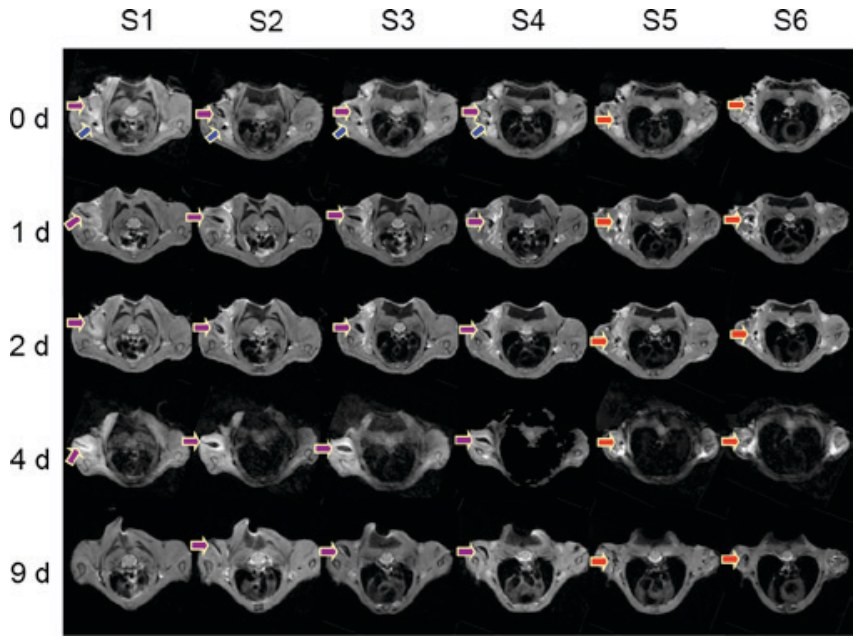

Figure 4. $T_{2}$-weighted magnetic resonance imaging of a mouse forelimb receiving injection of $\sim 2 \times 10^{5}$ live human mesenchymal stem cells labeled with (Gd-DOTA) $)_{4}$ TPP. d stands for day. S defines slice sequence. Signal reduction exhibits at the injection site, and persists over 9 days.

a signal enhancement effect in its surrounding tissue and is cleared/migrates away one day after cell transplantation. The cell clot indicated by red arrows in S5-S6 and 0-9 days of Fig. 4 also induces a signal enhancement effect in its surrounding tissue but is cleared/migrates away over a longer time with a gradual decrease in clot size from 0 to 4 days. These observations suggest that the in vivo fates and physiological activity of

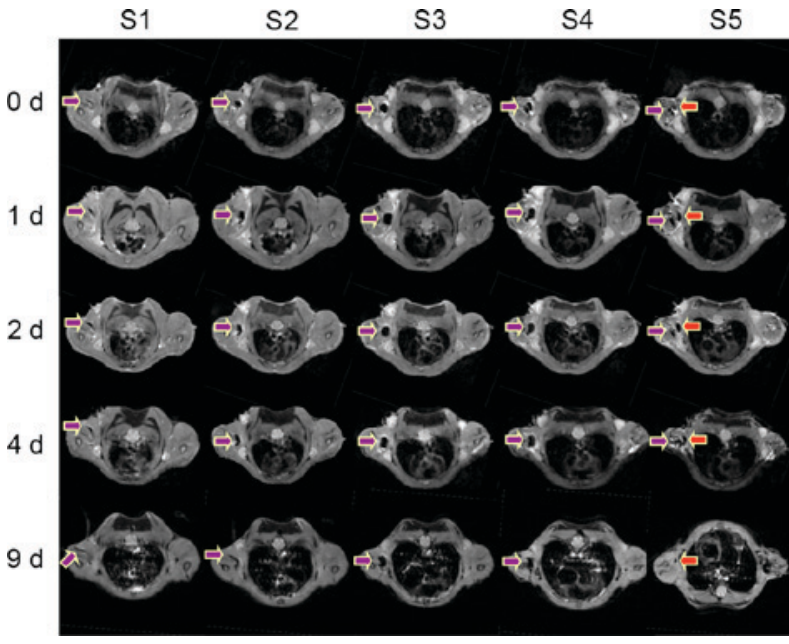

Figure 5. $\mathrm{T}_{2}$-weighted magnetic resonance imaging of a mouse forelimb receiving injection of $\sim 2 \times 10^{5}$ dead human mesenchymal stem cells labeled with (Gd-DOTA) $)_{4}$-TPP. d stands for day. S defines slice sequence. Signal reduction exhibits at the injection site, and persists over 9 days.

cell transplants are likely dependent on the nature of its host tissue.

In order to reveal the fates of the live cell transplant in the limb muscle, histological analysis were conducted on the muscular tissue with cell transplantation. The results are shown in Fig. 6 where nuclei of either live or apoptotic cells appear in blue with DAPI staining, and apoptotic cells appear in red with 
$0 \mathrm{~d}$

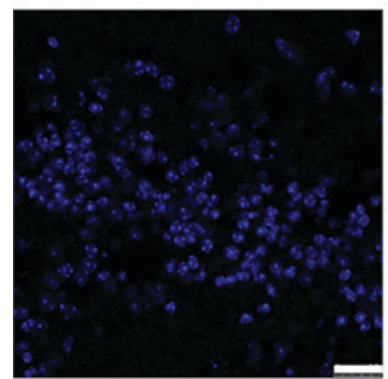

$4 d$

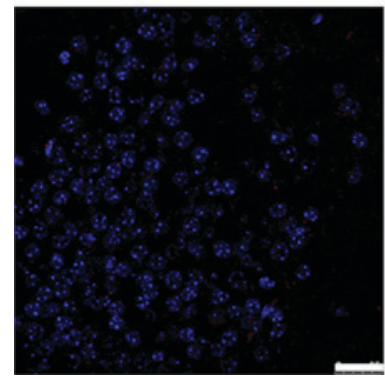

$1 d$

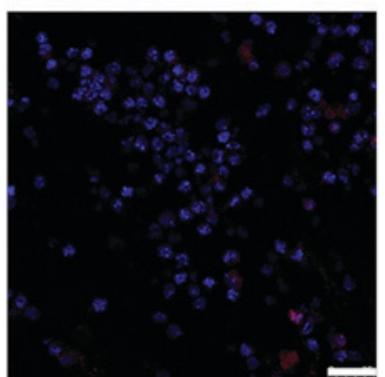

$7 d$

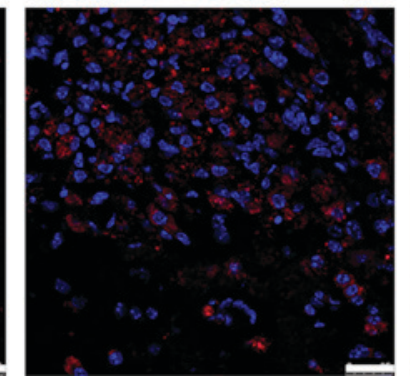

$2 d$

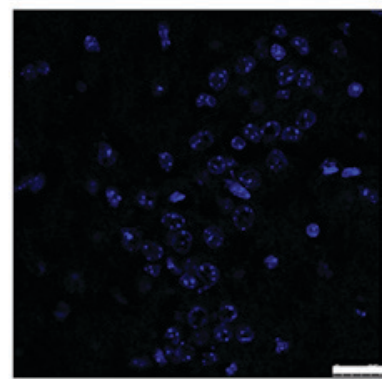

$10 \mathrm{~d}$

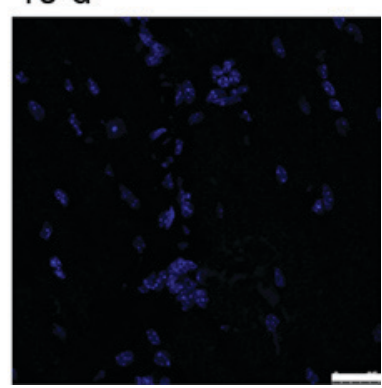

Figure 6. Histological analysis of mice muscular tissue with injection of $\sim 3 \times 10^{5}$ live hMSCs labeled with (Gd-DOTA) ${ }_{4}$-TPP as a function of post-injection time. d stands for day. Scale bar $=25 \mu \mathrm{m}$.

C-caspase-3 staining. No cell apoptosis is reported on 0 day; sporadic cell apoptosis exhibits from $1 \sim 4 \mathrm{~d}$ as indicated by the sporadic red cell nucleus; large scale cell apoptosis appears on $7 \mathrm{~d}$ as indicated by the large amount of red cell nucleus. The results suggest that large scale cell apoptosis occurs to the live cell transplants within one week. The histological results are in agreement with that of Zhang et al where large scale cell apoptosis was also reported on $4 \mathrm{~d}$ with cell staining (27). On the other hand, Ishikane et al reported that large number of GFP-positive MSCs could be detected on $7 \mathrm{~d}$ after transplantation into ischemic hindlimb muscle of a rat with a small number detectable even on $21 \mathrm{~d}$ after cell injection, suggesting a long period of post-injection cell survival (21). The discordance might be ascribed to different in vivo viability of the cell transplants. It is also possible that a slow in vivo clearance rate of GFP proteins released by dead cells may lead to overestimation of cell survival.

It has been observed in our intracranial cell transplantation that a significant signal enhancement appears in the surrounding tissue which can be ascribed to the free diffusion of small (Gd-DOTA) $)_{4}$-TPP molecules released as a result of exocytosis of live cells, and is thus associated with an active physiological activity of the live cells (16). This also occurs to the live cell transplants in mouse limb muscles where a signal enhancement effect exhibits in the right forelimb with respect to the left forelimb [Fig. 4 (S1-S6 and 1-4 days)], but is not so obvious for the dead cell transplants in mouse limb muscles (Fig. 5). In this respect, we also note that in the reported intracranial cell transplantation, an obvious and localized signal enhancement effect exhibits in the proximity or core of cell transplants on $4 \mathrm{~d}$ for dead cell injection or on $7 \mathrm{~d}$ after death of live cell transplants (16). Such a localized signal enhancement effect is absent in case of muscular transplantation till 9 $\mathrm{d}$ after either live or dead cell injection (Figs. 4 and 5). This is due likely to difference in the biological microenvironment between the limb muscle and the brain tissues or a difference in the clearance process of dead cells in between the limb muscular and the brain tissues.

The MRI results obtained so far from EP-labeling with (Gd-DOTA) $)_{4}$-TPP demonstrate that cell migration occurs to live cell transplants in hippocampus (16) and subcutaneous tissue but not in the cortex/corpus callosum (16) or limb muscles. No cell migration has been observed for dead cell transplants. Hence, cell migration away from the transplantation site can likely be taken as an indication of cell survival. Free diffusion of small (Gd-DOTA) $)_{4}$-TPP molecules released as a result of exocytosis of live cell transplants induces signal enhancement in the surrounding tissue, which can also be taken as an indication of physiological activity of live cells. However, it is still tricky to tell live cells from dead ones at the site of cell transplantation. Targeting cell migration and homing is yet to be demonstrated by our labeling and imaging strategy with appropriate animal models.

Our labeling and imaging strategy features single contrast agent, single imaging mode and dual signal output.

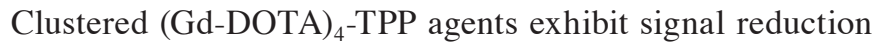
and slow clearance whereas free (Gd-DOTA) ${ }_{4}$-TPP agents exhibit signal enhancement and fast clearance. When clustered (Gd-DOTA) $)_{4}$-TPP agents are released as free agents, a signal reduction effect is switched to a signal enhancement effect. Such a signal switch combined with different rate of in vivo clearance of relevant agents can reveal more information associated with in vivo fates of labeled cell transplants compared with cell-labeling strategies with Gd agents for $\mathrm{T}_{1}$-weighted MRI $(9,15,23)$. In addition, it also avoids the introduction of any synthetic coating material into cells or tissues. This is an additional advantage over the use of conventional surface-coated nanoparticle-labeling $(28,29)$ in terms of biocompatibility, cytotoxicity, in vivo degradation and clearance. 
In summary, cell labeling with (Gd-DOTA) ${ }_{4}$-TPP via EP can significantly promote its intracellular retention time and also induces a significant signal reduction effect that favors $\mathrm{T}_{2}$-weighted MRI. The labeling and imaging strategy allows unambiguous distinguishing of cell transplants from their surrounding tissues and provide a novel tool for long term in vivo tracking of cell transplants. The MRI results reveal that the fates and physiological activities of cell transplants depend on the nature of its host tissues. A slow clearance process occurs to dead cell transplants in limb muscular tissue. Although it cannot report cell death at the cell transplantation site of muscular tissue, in vivo migration of labeled live cells can be unambiguously reported.

\section{Acknowledgements}

This study was funded by general projects from the Natural Science Foundation of China (nos. 21673281 and 31371010), a Strategic Priority Research Program of the Chinese Academy of Sciences (no. XDA01030203), and a Basic Research Project from the Ministry of Science and Technology of China (no. 2011CB965004).

\section{References}

1. Rogers WJ, Meyer CH and Kramer CM: Technology insight: In vivo cell tracking by use of MRI. Nat Clin Pract Cardiovasc Med 3: 554-562, 2006.

2. Kraitchman DL and Bulte JW: Imaging of stem cells using MRI. Basic Res Cardiol 103: 105-113, 2008.

3. Gera A, Steinberg GK and Guzman R: In vivo neural stem cell imaging: Current modalities and future directions. Regen Med 5: 73-86, 2010.

4. Mahmoudi M, Hosseinkhani H, Hosseinkhani M, Boutry S, Simchi A, Journeay WS, Subramani K and Laurent S: Magnetic resonance imaging tracking of stem cells in vivo using iron oxide nanoparticles as a tool for the advancement of clinical regenerative medicine. Chem Rev 111: 253-280, 2011.

5. Khurana A, Nejadnik H, Gawande R, Lin G, Lee S, Messing S, Castaneda R, Derugin N, Pisani L, Lue TF and Daldrup-Link HE: Intravenous ferumoxytol allows noninvasive MR imaging monitoring of macrophage migration into stem cell transplants. Radiology 264: 803-811, 2012.

6. Bulte JW: Science to practice: Can macrophage infiltration serve as a surrogate marker for stem cell viability? Radiology 264 619-620, 2012.

7. Nejadnik H, Ye D, Lenkov OD, Donig JS, Martin JE, Castillo R, Derugin N, Sennino B, Rao J and Daldrup-Link H: Magnetic resonance imaging of stem cell apoptosis in arthritic joints with a caspase activatable contrast agent. ACS Nano 9: 1150-1160, 2015.

8. Ngen EJ, Wang L, Kato Y, Krishnamachary B, Zhu W, Gandhi N, Smith B, Armour M, Wong J, Gabrielson K and Artemov D: Imaging transplanted stem cells in real time using an MRI dual-contrast method. Sci Rep 5: 13628, 2015.

9. Tachibana Y, Enmi JI, Mahara A, Iida H and Yamaoka T: Design and characterization of a polymeric MRI contrast agent based on PVA for in vivo living-cell tracking. Contrast Media Mol Imaging 5: 309-317, 2010.

10. Hoshino K, Ly HQ, Frangioni JV and Hajjar RJ: In vio tracking in cardiac stem cell-based therapy. Prog Cardiovasc Dis 49: 414-420, 2007.

11. Wang YX, Wang HH, Au DW, Zou BS and Teng LS: Pitfalls in employing superparamagnetic iron oxide particles for stem cell labeling and in vivo MRI tracking. Brit J Radiol 81: 987-988, 2008 .
12. Amsalem Y, Mardor Y, Feinberg MS, Landa N, Miller L, Daniels D, Ocherashvilli A, Holbova R, Yosef O, Barbash IM and Leor J: Iron-oxide labeling and outcome of transplanted mesenchymal stem cells in the infarcted myocardium. Circulation 116 (11 Suppl): I38-I45, 2007.

13. Li Z, Suzuki Y, Huang M, Cao F, Xie X, Connolly AJ, Yang PC and $\mathrm{Wu} \mathrm{JC}$ : Comparison of reporter gene and iron particle labeling for tracking fate of human embryonic stem cells and differentiated endothelial cells in living subjects. Stem Cells 26: 864-873, 2008

14. Terrovitis J, Stuber M, Youssef A, Preece S, Leppo M, Kizana E, Schär M, Gerstenblith G, Weiss RG, Marbán E and Abraham MR: Magnetic resonance imaging overestimates ferumoxide-labeled stem cell survival after transplantaion in the heart. Circulation 117: 1555-1562, 2008.

15. Agudelo CA, Tachibana Y, Hurtado AF, Ose T, Iida $\mathrm{H}$ and Yamaoka T: The use of magnetic resonance cell tracking to monitor endothelial progenitor cells in a rat hindlimb ischemic model. Biomaterials 33: 2439-2448, 2012.

16. Zhang YH, Zhang HY, Li BB, et al: Cell-assembled (Gd-DOTA) -TPP nanoclusters as T2 contrast agent reveal in vivo fates of stem cell transplants. Nano Res: 2017 (Epub ahead of print).

17. Shintani S, Murohara T, Ikeda H, Ueno T, Sasaki K, Duan J and Imaizumi T: Augmentation of postnatal neovascularization with autologous bone marrow transplantation. Circulation 103: 897-903, 2001.

18. Oswald J, Boxberger S, Jørgensen B, Feldmann S, Ehninger G, Bornhäuser $M$ and Werner $C$ : Mesenchymal stem cells can be differentiated into endothelial cells in vitro. Stem Cells 22: $377-384,2004$

19. Iwase T, Nagaya N, Fujii T, Itoh T, Murakami S, Matsumoto T, Kangawa $\mathrm{K}$ and Kitamura S: Comparison of angiogenic potency betweeen mesenchymal stem cells and mononuclear cells in a rat model of hindlimb ischemia. Cardiovas Res 66: 543-551, 2005.

20. Dai WD, Hale SL, Martin BJ, Kuang JQ, Dow JS, Wold LE and Kloner RA: Allogeneic mesenchymal stem cell transplantation in postinfarcted rat myocardium: Short- and long-term effects. Circulation 112: 214-223, 2005.

21. Ishikane S, Ohnishi S, Yamahara K, Sada M, Harada K, Mishima K, Iwasaki K, Fujiwara M, Kitamura S, Nagaya N and Ikeda T: Allogeneic injection of fetal membrane-derived mesenchymal stem cells induces therapeutic angiogenesis in a rat model of hind limb ischemia. Stem Cells 26: 2625-2633, 2008.

22. Wingate K, Bonani W, Tan Y, Bryant SJ and Tan W: Compressive elasticity of three-demensional nanofiber matrix directs mesenchymal stem cell differentiation to vascular cells with endothelial or smooth muscle cell markers. Acta Biomater 8: 1440-1449, 2012.

23. Tachibana Y, Enmi JI, Agudelo CA, Iida H and Yamaoka T: Long-term/bioinert labeling of rat mesenchymal stem cells with PVA-Gd conjugates and MRI monitoring of the labeled cell survival after intramuscular transplantation. Bioconj Chem 25: 1243-1251, 2014.

24. Murphy MP and Smith RAJ: Targeting antioxidants to mitochondria by conjugation to lipophilic cations. Ann Rev Pharmacol Toxic 47: 629-656, 2007.

25. Murphy MP and Smith RAJ: Drug delivery to mitochondria: The key to mitochondrial medicine. Adv Drug Del Rev 41: 235-250, 2000.

26. Wang $\mathrm{C}$, Cheng $\mathrm{L}, \mathrm{Xu} \mathrm{H}$ and Liu Z: Towards whole-body imaging at the single cell level using ultra-sensitive stem cell labeling with oligo-arginine modified upconversion nanoparticles. Biomaterials 33: 4872-4881, 2012.

27. Zhang M, Methot D, Poppa V, Fujio Y, Walsh K and Murry CE: Cardiomyocyte grafting for cardiac repair: Graft cell death and anti-death strategies. J Mol Cell Cardiol 33: 907-921, 2001.

28. Chen HW, Yeh J, Wang LY, Khurshid H, Peng N, Wang AY and Mao H: Preparation and control of th formation of single core and clustered nanoparticles for biomedical applications using a verstile amphiphilic diblock copolymer. Nano Res 3: 852-862, 2010.

29. Chen HW, Zou P, Connam J, Paholak H and Sun DX: Intracellular dissociation of a polymer coating from nanoparticles. Nano Res 5: 815-825, 2012. 